\title{
Influência do processo de familiarização para avaliação da força muscular em testes de 1-RM
}

Raphael Mendes Ritti Dias ${ }^{1}$, Edilson Serpeloni Cyrino ${ }^{1}$, Emanuel Péricles Salvador ${ }^{1}$, Lúcio Flávio Soares Caldeira1, Fábio Yuzo Nakamura', Rafael Raul Papst",

Nelson Bruna ${ }^{1}$ e André Luiz Demantova Gurjão ${ }^{1}$

\section{RESUMO}

Embora testes de uma repetição máxima (1-RM) sejam freqüentemente utilizados para a avaliação da força muscular, acredita-se que os resultados obtidos possam ser afetados pela falta de familiarização prévia, até mesmo em sujeitos com experiência em exercícios com pesos. Assim, o objetivo deste estudo foi investigar o impacto do processo de familiarização para avaliação da força muscular em testes de 1-RM. Para tanto, 21 homens (24,5 $\pm 3,8$ anos), aparentemente saudáveis, com experiência prévia de pelo menos seis meses em treinamento com pesos, foram submetidos a testes repetitivos de 1-RM nos exercícios supino em banco horizontal, agachamento e rosca direta de bíceps. Os testes foram executados em quatro sessões, intervaladas a cada 48-72 horas. Um número máximo de três tentativas, com intervalo de três a cinco minutos para recuperação, foi utilizado em cada exercício, nas quatro sessões de testagem. ANOVA para medidas repetidas, seguida pelo teste post hoc de Tukey, quando $p \leq 0,05$, foi utilizada para o tratamento dos dados. Aumentos significantes na força muscu$\operatorname{lar}(p \leq 0,01)$ foram encontrados nos três exercícios analisados entre a primeira e a quarta sessão de familiarização $(2,4 \%$ no supino em banco horizontal, 3,4\% no agachamento e 5,4\% na rosca direta de bíceps). Todavia, nenhuma diferença estatisticamente significante foi encontrada entre a segunda e a quarta sessão de familiarização na rosca direta de bíceps $(p>0,05)$, bem como entre a terceira e a quarta sessão no supino em banco horizontal e no agachamento ( $p$ $>0,05)$. Os resultados indicam que a falta de familiarização prévia com testes de 1-RM pode comprometer a avaliação da força muscular. Portanto, sugere-se, para avaliação mais acurada da força muscular mediante testes de 1-RM, a execução de duas a três sessões de familiarização em homens adultos com experiência em exercícios com pesos.

\section{RESUMEN}

\section{Influencia del proceso de familiarizacion para evaluación de la fuerza muscular en tests de 1-RM}

Embora la utilización de tests de una repetición máxima (1-RM) es frecuente para la evaluación de la fuerza muscular, se acredita que los resultados obtenidos puedan ser afectados por la falta de familiarización previa, hasta mismo en sujetos con experiencia en ejercicios con pesos. Asi, el objetivo de este estudio fué investigar el impacto del proceso de familiarización para evaluación de la fuerza

\footnotetext{
1. Grupo de Estudo e Pesquisa em Metabolismo, Nutrição e Exercício, Centro de Educação Física e Desportos - Universidade Estadual de Londrina.
}

Recebido em 19/7/04. 2a versão recebida em 22/11/04. Aceito em 20/12/04.

Endereço para correspondência: Grupo de Estudo e Pesquisa em Metabolismo, Nutrição e Exercício, Centro de Educação Física e Desportos, Universidade Estadual de Londrina, Rod. Celso Garcia Cid, km 380 - Campus Universitário - 86051-990 - Londrina, PR - Brasil. E-mail: dias.raphael @uol.com.br
Palavras-chave: Força muscular. Exercícios com pesos. Adaptações neurais. Testes de 1-RM. Familiarização.

Palabras-clave: Fuerza muscular. Ejercicios con pesos. Adaptaciones neurales. Tests de 1-RM. Familiarización.

muscular en tests de 1-RM. Por lo tanto, 21 hombres $(24,5 \pm 3,8$ años), aparentemente saludables con experiencia previa de por lo menos seis meses de entrenamiento con pesos, fueron sometidos a tests reptitivos de 1-RM en los ejercicios supino en banco horizontal, agachamiento y rosca directa de bíceps. Los tests fueran ejecutados en cuatro sesiones, intervaladas en cada 48-72 horas. Un número máximo de tres tentativas, con intérvalo de tres a cinco minutos para recuperación, fué utilizado en cada ejercicio, en las cuatro sessiones de testeo. ANOVA se utilizó para medidas repetidas, seguida por el test post hoc de Tukey, cuando $\mathrm{p} \leq 0,05$, fué utilizada para el tratamiento de los datos. Aumentos significantes en la fuerza muscular ( $\mathrm{p} \leq 0,01$ ) fueron encontrados los tres ejercicios analizados entre la primera y la cuarta sesión de familiarización $2,4 \%$ en supino en banco horizontal, 3,4\% en agachamiento y 5,4\% en la rosca directa del bíceps). Todavía, ninguna diferencia estadísticamente significativamente fuera encontrada entre la segunda y la cuarta sesión de familiarización en la rosca directa de bíceps ( $\mathrm{p}>0,05)$, bien con entre la tercera y la cuarta sesión en el supino en el banco horizontal y en el agachamiento ( $\mathrm{p}$ $>0,05)$. Los resultados indican que la falta de familiarización previa con tests de 1-RM puede comprometer la evaluación de la fuerza muscular. Por lo tanto, se sugiere para la evaluación mas acurada de la fuerza muscular mediante tests de 1-RM la ejecución de dos a tres sesiones de familiarización en los hombres adultos con experiencia en ejercicios con pesos.

\section{INTRODUÇÃO}

A força muscular é um importante componente da aptidão física relacionada à saúde, além de exercer papel relevante para o desempenho físico em inúmeras modalidades esportivas. Dentre as diferentes formas de treinamento para o desenvolvimento da força muscular, destaca-se a prática de exercícios com pesos.

Nesse sentido, aumentos significantes nos níveis de força muscular podem ser observados mediante a aplicação de testes específicos já nas primeiras semanas de treinamento com pesos (TP), em sujeitos de ambos os sexos, de diferentes faixas etárias e níveis de aptidão física variados ${ }^{(1-6)}$.

De acordo com a literatura, verifica-se que o teste de uma repetição máxima (1-RM) vem sendo o mais freqüentemente utilizado para avaliação da força dinâmica, sobretudo por pesquisadores e profissionais das áreas do exercício físico e do esporte, uma vez que é um método prático, de baixo custo operacional e aparentemente seguro para a maioria das populações.

Contudo, estudos recentes têm indicado aumentos significantes na força muscular em testes repetitivos de 1-RM em homens e mulheres jovens, bem como em mulheres idosas, sem expe- 
riência prévia ou recente em exercícios com pesos ${ }^{(7-9)}$. Esses resultados indicam que a falta de familiarização com os procedimentos que envolvem a execução de testes de 1-RM podem comprometer a análise das informações, principalmente em estudos de acompanhamento.

Por outro lado, nenhuma informação adicional tem sido produzida pela literatura ao longo das últimas três décadas com relação ao comportamento de sujeitos com experiência prévia em exercícios com pesos durante testes repetitivos de 1-RM. Assim, acredita-se que o processo de familiarização nesses indivíduos possa ser mais curto do que aquele observado em indivíduos inexperientes com esse tipo de exercício físico.

Desse modo, o objetivo deste estudo foi verificar o número de sessões necessárias para a familiarização em testes de 1-RM, em homens fisicamente ativos, com experiência prévia em exercícios com pesos.

\section{METODOLOGIA}

\section{Sujeitos}

Vinte e um homens $(24,5 \pm 3,8$ anos), aparentemente saudáveis, participaram voluntariamente deste estudo. Como critérios iniciais de inclusão, os participantes deveriam ser fisicamente ativos (atividade física regular sistematizada > duas vezes por semana) além de ter experiência de pelo menos seis meses em TP. Os sujeitos, após serem previamente esclarecidos sobre os propósitos da investigação e procedimentos aos quais seriam submetidos, assinaram um termo de consentimento livre e esclarecido. Este estudo foi aprovado pelo Comitê de Ética em Pesquisa da Universidade Estadual de Londrina, de acordo com as normas da Resolução 196/96 do Conselho Nacional de Saúde sobre pesquisa envolvendo seres humanos.

\section{Antropometria}

A massa corporal foi mensurada em uma balança de plataforma, digital, marca Urano, modelo PS 180, com precisão de 0,1 kg, e a estatura foi obtida em um estadiômetro de madeira com precisão de 0,1 cm, de acordo com os procedimentos descritos por Gordon et al. ${ }^{(10)}$. Todos os indivíduos foram medidos e pesados descalços, vestindo apenas uma sunga. A partir dessas medidas, o índice de massa corporal (IMC) foi determinado pelo quociente massa corporal/estatura ${ }^{2}$, sendo a massa corporal expressa em quilogramas $(\mathrm{kg})$ e a estatura, em metros (m).

A composição corporal foi determinada pela técnica de espessura de dobras cutâneas. Três medidas foram tomadas em cada ponto anatômico (abdômen, supra-ilíaca, axilar-média, subescapular, tricipital, peitoral e coxa), em seqüência rotacional, do lado direito do corpo, sendo registrado o valor mediano. Tais medidas foram realizadas por um único avaliador com um adipômetro científico da marca Lange, de acordo com as técnicas descritas por Slaughter et al.(11). O coeficiente teste-reteste excedeu 0,95 para cada um dos pontos anatômicos, com erro técnico de medida de no máximo $\pm 1,0 \mathrm{~mm}$.

A gordura corporal relativa (\% gordura) foi calculada pela fórmula de Siri ${ }^{(12)}$, a partir da estimativa da densidade corporal determinada pela equação envolvendo a espessura de sete dobras cutâneas proposta por Jackson e Pollock ${ }^{(13)}$

\section{Força muscular}

Todos os sujeitos foram submetidos a quatro sessões de testes de 1-RM, nos exercícios supino em banco horizontal, agachamento e rosca direta de bíceps, nessa ordem respectivamente, com intervalo de 48 a 72 horas entre cada sessão, para avaliação da força muscular.

Cada um dos três exercícios foi precedido por uma série de aquecimento (6 a 10 repetições), com aproximadamente 50\% da carga a ser utilizada na primeira tentativa de cada teste de 1-RM. A testa- gem foi iniciada dois minutos após o aquecimento. Os sujeitos foram orientados para tentar completar duas repetições. Caso fossem completadas duas repetições na primeira tentativa, ou mesmo se não fosse completada sequer uma repetição, uma segunda tentativa era executada após um intervalo de recuperação de três a cinco minutos com uma carga superior (primeira possibilidade) ou inferior (segunda possibilidade) àquela empregada na tentativa anterior. Tal procedimento foi repetido novamente em uma terceira e derradeira tentativa, caso ainda não se tivesse determinado a carga referente a uma única repetição máxima. Portanto, a carga registrada como 1-RM foi aquela na qual foi possível ao indivíduo completar somente uma única repetição(14). O intervalo de transição entre os exercícios foi de três a cinco minutos.

Vale ressaltar que a forma e a técnica de execução de cada exercício foram padronizadas e continuamente monitoradas na tentativa de garantir a qualidade das informações. Além disso, os sujeitos realizaram os testes sempre no mesmo período do dia (manhã, tarde ou noite) e não praticaram exercícios físicos durante o período experimental.

\section{Tratamento estatístico}

Inicialmente, os dados foram tratados a partir de procedimentos descritivos, com as informações sendo processadas no pacote computacional Statistica, versão 5.1. Análise de variância (ANOVA) para medidas repetidas foi utilizada para as comparações entre os escores obtidos nos testes de 1-RM executados em diferentes sessões, nos exercícios supino em banco horizontal, agachamento e rosca direta de bíceps. O teste post hoc de Tukey foi empregado para a identificação das diferenças específicas nas variáveis em que os valores de $\mathrm{F}$ encontrados foram superiores ao do critério de significância estatística estabelecido $(p \leq 0,05)$. O limite de concordância entre as sessões de familiarização em que ocorreu a suposta estabilização da força muscular, em cada um dos três exercícios investigados, foi analisado mediante os procedimentos propostos por Bland e Altman ${ }^{(15)}$.

\section{RESULTADOS}

A tabela 1 apresenta a descrição das características físicas dos sujeitos investigados.

TABELA 1

Características físicas dos sujeitos $(\mathrm{n}=\mathbf{2 1}$ )

\begin{tabular}{lrrrr}
\hline & Média & DP & Mínimo & Máximo \\
& 24,5 & 3,8 & 20,0 & 36,0 \\
Idade (anos) & 74,0 & 10,6 & 62,4 & 103,9 \\
Massa corporal $(\mathrm{kg})$ & 175,1 & 6,6 & 156,5 & 189,0 \\
Estatura (cm) & 24,1 & 2,6 & 20,9 & 29,1 \\
IMC (kg/m $\left.{ }^{2}\right)$ & 12,5 & 3,4 & 5,8 & 19,2 \\
\% Gordura & 64,5 & 6,7 & 57,1 & 83,9 \\
Massa corporal magra (kg) & 9,5 & 4,0 & 3,6 & 20,0 \\
Massa gorda (kg) & & & & \\
\hline
\end{tabular}

Na tabela 2 encontram-se os valores de 1-RM obtidos nas diferentes sessões de testes, nos três exercícios executados. Nos exercícios supino em banco horizontal e agachamento, a estabilização da carga referente a 1-RM ocorreu apenas entre as sessões três e quatro, ao passo que no exercício rosca direta de bíceps, ANOVA indicou estabilização da força muscular a partir da segunda sessão. Em termos percentuais, a evolução de 1-RM entre a primeira sessão e a sessão associada à estabilização da força muscular foi equivalente a 1,9\% para o supino em banco horizontal, $2,4 \%$ para o agachamento e 3,4\% para a rosca direta de bíceps.

As figuras 1, 2 e 3 apresentam as plotagens propostas por Bland e Altman ${ }^{(15)}$ para verificação de concordância entre as medidas obtidas nas sessões nas quais se configurou estatisticamente o processo de estabilização das cargas. Nas figuras 1 e 2 estão plotadas as diferenças individuais (eixo y) de 1-RM entre as sessões 
três e quatro em função das médias entre as duas sessões (eixo x) para os exercícios supino em banco horizontal e agachamento, respectivamente. O mesmo tipo de representação gráfica foi utilizado para expressar a concordância entre as sessões dois e três para o exercício rosca direta de bíceps (figura 3).

\section{TABELA 2}

Força muscular de homens adultos com experiência em exercícios com pesos durante testes repetitivos de 1-RM $(n=21)$

\begin{tabular}{llll}
\hline \multicolumn{1}{c}{ Exercícios } & Média & DP & Amplitude \\
\hline Supino em banco horizontal (kg) & & & \\
Sessão 1 & 74,6 & 14,4 & $47,1-103,1$ \\
Sessão 2 & $75,7^{\mathrm{a}}$ & 14,6 & $47,1-105,1$ \\
Sessão 3 & $76,0^{\mathrm{a}}$ & 14,7 & $47,1-107,1$ \\
Sessão 4 & $76,4^{\mathrm{a}, \mathrm{b}}$ & 14,7 & $47,1-109,1$ \\
\hline Agachamento $(\mathrm{kg})$ & & & \\
Sessão 1 & 147,0 & 25,1 & $112,3-208,3$ \\
Sessão 2 & $149,6^{\mathrm{a}}$ & 23,9 & $116,3-208,3$ \\
Sessão 3 & $150,5^{\mathrm{a}}$ & 24,5 & $116,3-208,3$ \\
Sessão 4 & $151,9^{\mathrm{a}, \mathrm{b}}$ & 24,9 & $116,3-208,3$ \\
\hline Rosca direta de bíceps (kg) & & & \\
Sessão 1 & 44,0 & 7,4 & $31,5-51,5$ \\
Sessão 2 & $45,5^{\mathrm{a}}$ & 7,3 & $33,5-57,5$ \\
Sessão 3 & $46,0^{\mathrm{a}}$ & 7,1 & $33,5-57,5$ \\
Sessão 4 & $46,4^{\mathrm{a}}$ & 7,1 & $35,5-57,5$ \\
\hline
\end{tabular}

a $p \leq 0,01$ vs. sessão $1 \mathrm{e}^{\mathrm{b}} p<0,05$ vs. sessão 2 .

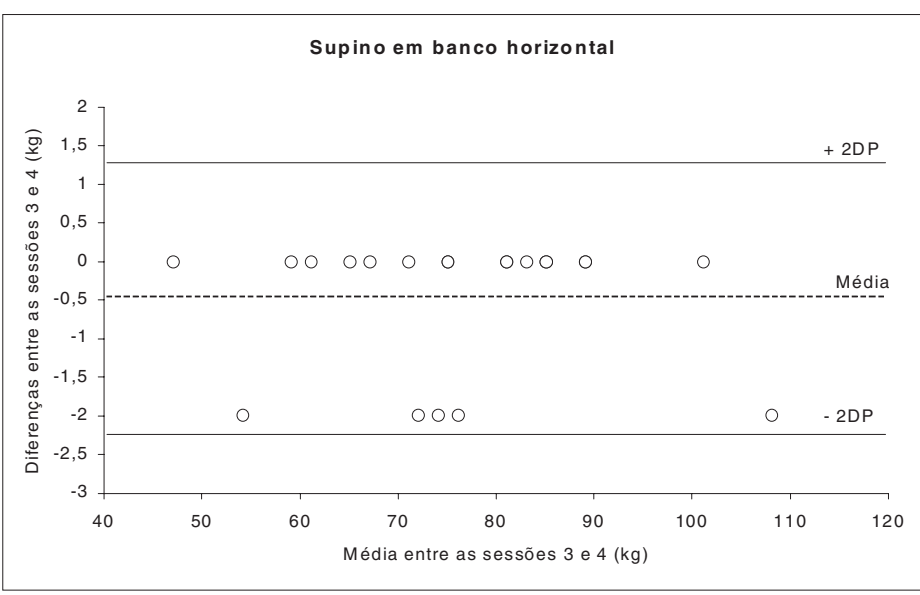

Fig. 1 - Plotagem de Bland-Altman para comparações entre as sessões três e quatro de testes de 1-RM para o exercício supino em banco horizontal $(n=21)$

Nota - Quatro pontos estão sobrepostos

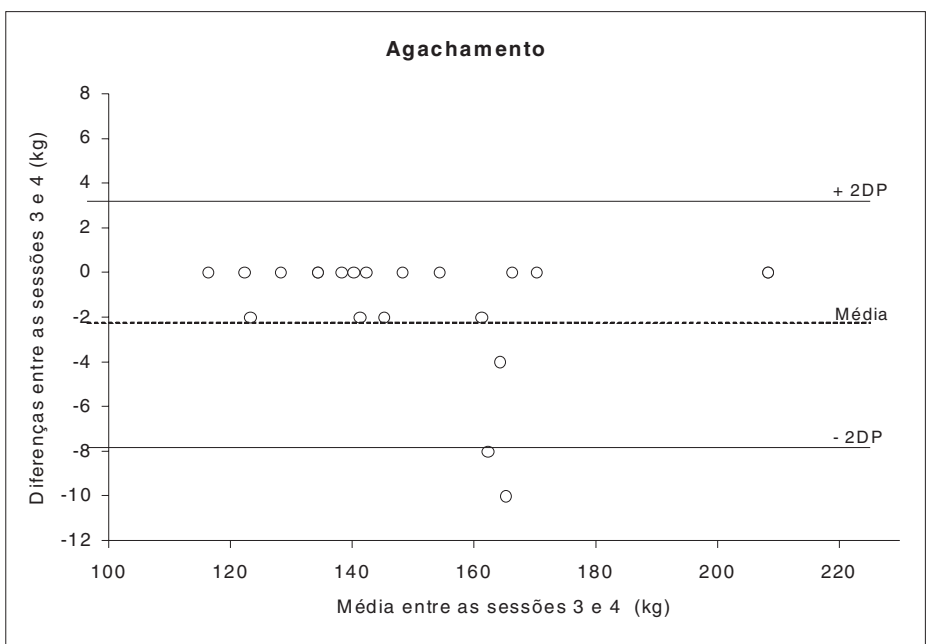

Fig. 2 - Plotagem de Bland-Altman para comparações entre as sessões três e quatro de testes de 1-RM para o exercício agachamento $(n=21)$ Nota - Dois pontos estão sobrepostos

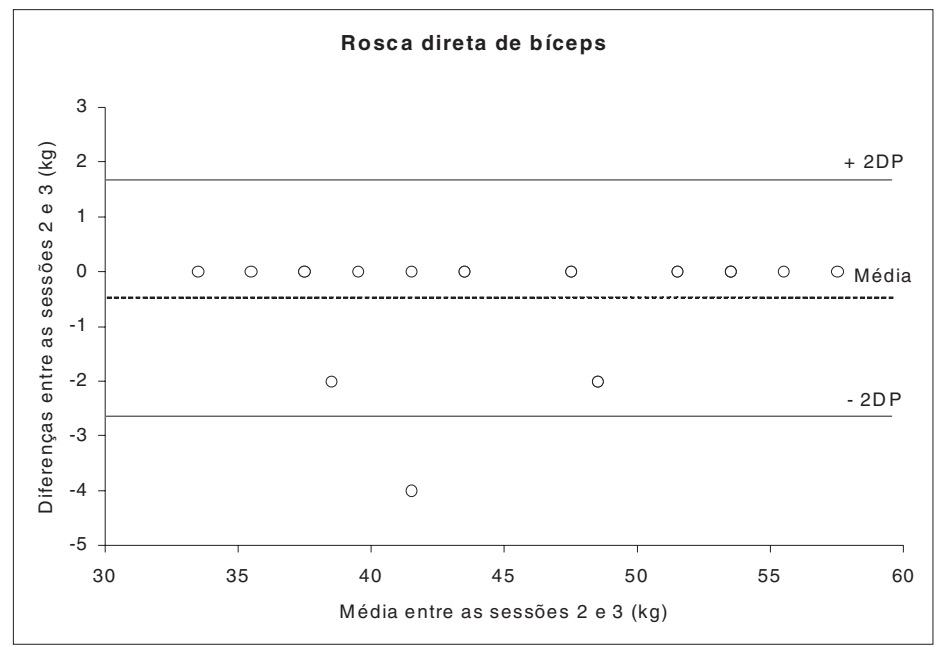

Fig. 3 - Plotagem de Bland-Altman para comparações entre as sessões dois e três de testes de 1-RM para o exercício rosca direta de bíceps ( $n=$ 21)

Nota - Sete pontos estão sobrepostos

Verificou-se em todos os exercícios estudados que a diferença média (linha central) e o espaço compreendido entre os limites de concordância (linhas superior e inferior) foram relativamente pequenos, sobretudo quando analisados com base em outras comparações pareadas (tabela 3).

TABELA 3

Média das diferenças e intervalo de confiança $(95 \%)$ entre os pares de avaliações da força máxima por meio de teste de 1-RM $(n=21)$

\begin{tabular}{lrcccc}
\hline Supino em banco horizontal & \multicolumn{3}{c}{ Sessões } & Estabilização \\
\cline { 2 - 5 } & $\mathbf{1 - 2}$ & $\mathbf{1 - 3}$ & $\mathbf{1 - 4}$ & $\mathbf{3 - 4}$ \\
Média das diferenças & $-1,0$ & $-1,3$ & $-1,8$ & $-0,5$ \\
Intervalo de confiança & 5,3 & 6,3 & 7,3 & 3,4 \\
\hline Agachamento & \multicolumn{3}{c}{ Sessões } & Estabilização \\
\cline { 2 - 5 } & $\mathbf{1 - 2}$ & $\mathbf{1 - 3}$ & $\mathbf{1 - 4}$ & $\mathbf{3 - 4}$ \\
Média das diferenças & $-2,7$ & $-3,5$ & $-5,0$ & $-1,4$ \\
Intervalo de confiança & 12,7 & 16,0 & 22,7 & 10,9 \\
\hline Rosca direta de bíceps & \multicolumn{3}{c}{ Sessões } \\
\cline { 2 - 5 } & $\mathbf{1 - 2}$ & $\mathbf{1 - 3}$ & $\mathbf{1 - 4}$ & Estabilização \\
Média das diferenças & $-1,5$ & $-2,0$ & $-2,4$ & $\mathbf{2 - 3}$ \\
Intervalo de confiança & 5,5 & 7,0 & 8,4 & $-0,5$ \\
\hline
\end{tabular}

Os resultados da tabela 3 demonstram que tanto a diferença média quanto o intervalo de confiança, compreendido entre os limites de concordância, foram reduzidos nas sessões em que ocorreu a estabilização da 1-RM, quando comparados com os outros pares de tentativas. Vale ressaltar que esse padrão foi observado em todos os exercícios investigados.

\section{DISCUSSÃO}

Os resultados deste estudo indicaram aumentos significantes na força muscular entre diferentes sessões de testes de 1-RM nos exercícios supino em banco horizontal, agachamento e rosca direta de bíceps, em sujeitos com experiência prévia em TP.

Esses achados são bastante interessantes, uma vez que a grande maioria dos estudos disponíveis na literatura que têm utilizado testes de 1-RM para a avaliação dos níveis de força muscular não trazem informações sobre a adoção de procedimentos de familia- 
rização prévia dos sujeitos com esses tipos de testes, em seus delineamentos experimentais. Dessa forma, a interpretação dos resultados obtidos por esses estudos pode ser bastante comprometida.

Embora alguns autores relatem índices de confiabilidade moderados ou, até mesmo, elevados, de acordo com o coeficiente de correlação intraclasses para o teste de 1-RM(7,8,16-18), os resultados devem ser analisados com certa cautela, visto que no presente estudo, apesar de os coeficientes teste e reteste entre a primeira e a quarta sessão serem elevados (supino em banco horizontal, $r=$ 0,96; agachamento, $r=0,98$; rosca direta de bíceps, $r=0,98$ ), diferenças estatisticamente significantes entre as cargas levantadas foram encontradas nos três exercícios investigados na comparação entre essas duas sessões.

Assim, acredita-se que a utilização de análise de correlação intraclasses entre as medidas não seria o procedimento mais adequado nessas situações, visto que esse índice não parece suficientemente sensível para analisar as modificações individuais ${ }^{(19)}$.

Nesse sentido, a plotagem de Bland e Altman ${ }^{(15)}$ foi utilizada no presente estudo por se tratar de recurso estatístico que permite a análise da concordância entre as medidas obtidas nas diferentes sessões de testes de 1-RM. Os resultados encontrados possibilitaram a confirmação dos momentos em que foi verificada estabilização das cargas, nos três exercícios estudados (figuras 1, 2 e 3).

Vale destacar que os valores referentes ao intervalo de confiança entre os exercícios foram menores nas sessões de estabilização quando confrontados com os outros pares de avaliações da força muscular (tabela 3). Portanto, um padrão temporal parece ser estabelecido quando os limites de concordância são adotados como referência para identificação da ocorrência de estabilização em testes de 1-RM.

A importância de sessões de familiarização para a obtenção de resultados mais acurados no teste de 1-RM vem sendo reportada recentemente por outros estudos ${ }^{(7-9)}$. Em um desses estudos, Ploutz-Snyder e Giamis ${ }^{(8)}$, ao analisarem o processo de familiarização com o teste de 1-RM, encontraram aumentos significantes na força muscular $(p<0,05)$, tanto em mulheres jovens $(12 \% ; n=7)$ quanto em idosas ( $22 \%$; $n=6)$. Entretanto, a quantidade de sessões de familiarização necessárias para a estabilização das cargas foi menor nas mulheres jovens quando comparadas com as idosas (3-4 e 8-9 sessões, respectivamente). Esses números foram superiores aos encontrados no presente estudo, que indicou a necessidade de apenas duas sessões de testes de 1-RM para o exercício rosca direta e três sessões para os exercícios supino em banco horizontal e agachamento.

As diferenças entre o presente trabalho e o de Ploutz-Snyder e Giamis $^{(8)}$ podem ser atribuídas, pelo menos em parte, aos tipos de exercícios e equipamentos utilizados, ao sexo e aos diferentes níveis de treinabilidade das amostras utilizadas nos dois estudos, uma vez que este investigou somente homens jovens, com experiência prévia em exercícios com pesos

Os resultados encontrados neste estudo sugerem que o processo de familiarização com o teste de 1-RM é importante não somente para indivíduos inexperientes em exercícios com pesos ${ }^{(7,8)}$, mas também para indivíduos com experiência nesse tipo de exercício físico.

Embora ainda não existam informações consistentes sobre os mecanismos responsáveis pelo aumento da força muscular em testes repetitivos de 1-RM, acredita-se que os mecanismos envolvidos sejam semelhantes àqueles verificados nas sessões iniciais de TP, tais como: aumento do recrutamento de unidades motoras de alto limiar, melhoria da coordenação dos grupos musculares antagonistas, aumento da freqüência de estimulação, melhoria na sincronização das unidades motoras estimuladas, dentre outros, denominados pela literatura de adaptações neurais ${ }^{(20-24)}$
Um desses mecanismos, a co-ativação dos músculos antagonistas ao movimento executado, foi confirmado por Carolan e Cafarelli(25), que verificaram, após oito semanas de TP, aumentos significantes na força de extensão de joelhos concomitantemente com a redução da co-ativação dos músculos antagonistas (flexores do joelho). Vale destacar que as maiores reduções na co-ativação dos músculos antagonistas ocorreram na primeira semana de treinamento.

Outro possível mecanismo neural envolvido nos aumentos iniciais de força muscular, o aumento da ativação da musculatura agonista, foi investigado por Häkkinen et al.(26). Os autores relataram aumentos estatisticamente significantes no sinal eletromiográfico dos extensores do joelho, tanto em homens quanto em mulheres, durante as duas primeiras semanas de TP, independente da faixa etária e dos níveis de treinabilidade.

Os resultados deste estudo indicaram, ainda, um padrão temporal semelhante para a familiarização com o teste de 1-RM nos exercícios envolvendo grandes grupos musculares (supino em banco horizontal e agachamento), diferente daquele observado no exercício rosca direta de bíceps, que envolve grupos musculares menores. Esses resultados vão de encontro aos achados de Cronin e Henderson ${ }^{(7)}$, que verificaram diferenças temporais para a familiarização com o teste de 1-RM nos exercícios supino em banco horizontal e agachamento, o que, segundo os autores, pode ser atribuído às diferenças no tamanho dos grupos musculares agonistas envolvidos na execução de cada um desses exercícios ou, ainda, aos diferentes níveis de complexidade das técnicas de execução. Considerando que a amostra do estudo foi composta exclusivamente por homens jovens ( $n=10 ; 21,0 \pm 2,7$ anos), com histórico atlético, porém sem prática de exercícios com pesos havia pelo menos seis meses, parece que a falta de experiência específica dos sujeitos também pode ter influenciado no padrão temporal de familiarização ao teste de 1-RM, nos exercícios investigados.

Vale ressaltar que os exercícios com pesos empregados neste estudo são freqüentemente utilizados nos diferentes programas de TP. Assim, a experiência prévia dos sujeitos na execução dos exercícios utilizados pode ter contribuído para que o processo de familiarização com os testes de 1-RM acontecesse mais rapidamente. Provavelmente, se os exercícios utilizados não fossem tão comuns aos sujeitos investigados, as respostas poderiam ter sido diferenciadas. Infelizmente, essa hipótese não foi testada no presente estudo, merecendo, portanto, futuras investigações.

\section{CONCLUSÃO}

As informações obtidas neste estudo indicam que a familiarização prévia com testes de 1-RM é de extrema importância para a análise da força muscular, também, em indivíduos com experiência prévia em exercícios com pesos. Todavia, parece que o padrão temporal do processo de familiarização é inferior ao verificado anteriormente por outros estudos, em sujeitos inexperientes com esse tipo de exercício físico. Além disso, quando os exercícios a serem testados envolvem grandes grupos musculares, parece ser necessário um período maior de familiarização quando comparado aos exercícios para pequenos grupos.

Os resultados sugerem que, para uma avaliação adequada da força muscular de homens adultos jovens, com experiência prévia em exercícios com pesos, sejam realizadas pelo menos três sessões de testes de familiarização em testes de 1-RM para os exercícios supino em banco horizontal e agachamento e duas sessões para o exercício rosca direta de bíceps.

Todos os autores declararam não haver qualquer potencial conflito de interesses referente a este artigo. 


\section{REFERÊNCIAS}

1. Anderson T, Kearney JT. Effects of three resistance training programs on muscular strength and absolute and relative endurance. Res Q Exerc Sport 1982;53: $1-7$.

2. Baker D, Wilson G, Carlyon R. Periodization: effect on strength of manipulating volume and intensity. J Strength Cond Res 1994;8:235-42.

3. Chestnut JL, Docherty D. The effects of 4 and 10 repetition maximum weighttraining protocols on neuromuscular adaptations in untrained men. J Strength Cond Res 1999;13:353-9.

4. Häkkinen K, Alen M, Komi PV. Changes in isometric force and relaxation-time, electromyographic and muscle fibre characteristics of human skeletal muscle during strength training and detraining. Acta Physiol Scand 1985;125:573-85.

5. Häkkinen KA, Pakarinen A, Alen M, Kauhanen H, Komi PV. Relationship between training volume, physical performance capacity, and serum hormone concentrations during prolonged training in elite weight lifters. Int J Sports Med 1987;8: $61-5$.

6. Kraemer JB, Stone MH, O'Bryant HS, Conley MS, Johnson RL, Nieman DC, et al. Effects of single vs. multiple sets of weight training: impact of volume, intensity, and variation. J Strength Cond Res 1997;11:143-7.

7. Cronin JB, Henderson ME. Maximal strength and power assessment in novice weight trainers. J Strength Cond Res 2004;18:48-52.

8. Ploutz-Snyder LL, Giamis EL. Orientation and familiarization to 1 RM strength testing in old and young women. J Strength Cond Res 2001;15:519-23.

9. Salvador EP, Dias RMR, Braghin RS, Cyrino ES. Influência da experiência em treinamento com pesos no processo de familiarização ao teste de 1-RM. In: III Congresso Científico Latino-Americano de Educação Física, Piracicaba, Unimep, $2004 ; 1159$.

10. Gordon CC, Chumlea WC, Roche AF. Stature, recumbent length, and weight. In: Lohman TG, Roche AF, Martorell R, editors. Anthropometric standardization reference manual. Champaign: Human Kinetics Books, 1988;3-8.

11. Slaughter MH, Lohman TG, Boileau RA, Stillman RJ, Van Loan M, Horswill CA, et al. Influence of maturation on relationship of skinfolds to body density: a cross-sectional study. Hum Biol 1984;56:681-9.

12. Siri WE. Body composition from fluid spaces and density: analysis of methods. In: Brozek J, Henschel A, editors. Techniques for measuring body composition. Washington: National Academy of Science, 1961;223-44.
13. Jackson AS, Pollock ML. Generalized equations for predicting body density of men. Br J Nutr 1978;40:497-504.

14. Clarke DH. Adaptations in strength and muscular endurance resulting from exercise. Exer Sports Sci Rev 1973;1:73-102.

15. Bland JM, Altman DJ. Regression analysis. Lancet 1986;1:908-9.

16. Pereira MIR, Gomes PSC. Testes de força e resistência muscular: confiabilidade e predição de uma repetição máxima - Revisão e novas evidências. Rev Bras Med Esporte 2003;9:325-35.

17. Rikli RE, Jones CJ, Beam WC, Duncan SJ, Lamar B. Testing versus training effects on 1-RM strength assessment in older adults. Med Sci Sports Exerc 1996;28:S153.

18. Braith RW, Graves JE, Leggett SH, Pollock ML. Effect of training on the relationship between maximal and submaximal strength. Med Sci Sports Exerc 1993; 25:132-8.

19. Vincent WJ. Statistics in kinesiology. Champaign: Human Kinetics Books, 1999.

20. Komi PV. Training of muscle strength and power: interaction of neuromotoric, hypertrophic, and mechanical factors. Int J Sports Med 1986;7(Suppl 1):10-5.

21. Enoka RM. Neural adaptations with chronic physical activity. J Biomech 1997; 30:447-55.

22. Sale DG. Neural adaptation to strength training. In: Komi PV, editor. Strength and power training. Oxford: Blackwell Scientific, 1992;249-65.

23. Moritani T, Devries HA. Neural factors versus hypertrophy in the time course of muscle strength gains. Am J Phys Med 1979;58:115-30.

24. Narici MV, Roi GS, Landoni L, Minetti AE, Cerretelli P. Changes in force, crosssectional area and neural activation during strength training and detraining of the human quadriceps. Eur J Appl Physiol 1989;59:310-9.

25. Carolan B, Cafarelli E. Adaptations in coactivation after isometric resistance training. J Appl Physiol 1992;73:911-7.

26. Häkkinen $K$, Kallinen $M$, Izquierdo $M$, Jokelainen $K$, Lassila $H$, Malkia $E$, et al. Changes in agonist-antagonist EMG, muscle CSA, and force during strength training in middle-aged and older people. J Appl Physiol 1998;84:1341-9. 